\title{
EL ROL DE LOS REQUERIMIENTOS DE CAPITAL EN EL MERCADO
} INTERBANCARIO

\author{
THE ROLE OF CAPITAL REQUIREMENTS IN THE INTERBANK \\ MARKET
}

Demian Macedo, ${ }^{1^{\star}}$ Audrone Virbickaite ${ }^{1}$

Departamento de Economía de la Empresa. Universidad de las Islas Baleares, España.

demian.macedo@uib.es; audrone.virbickaite@uib.es

RESUMEN

ABSTRACT

En este trabajo analizamos la capacidad de los requerimientos mínimos de capital para restaurar la provisión de liquidez en el mercado interbancario bajo un contexto de riesgo moral. En particular, estudiamos la efectividad de este tipo de regulación cuando las necesidades de liquidez pueden ser originadas por un shock de depósitos -contracción en la oferta de depósitos- o un shock de caja -incremento en préstamos calificados como dudosos-. La metodología aplicada en este trabajo se basa en el modelo de Macedo \& Troster (2019), el cual es una útil herramienta para analizar el comportamiento de la industria bancaria. Aquí extendemos dicho trabajo introduciendo capital regulatorio y estudiamos la asignación de liquidez del mercado interbancario ante diferentes shocks de liquidez. La conclusión evidencia que los requerimientos de capital son una herramienta útil para disminuir el riesgo moral en la industria bancaria y, de esta manera, restablecer el comercio interbancario. Además, mostramos que este tipo de regulación es más efectiva para tratar un shock de caja que un shock de depósito. Nuestro análisis es relevante para el logro de la estabilidad financiera, ya que permite entender como los requerimientos de capital operan en el mercado interbancario.

In this paper we analyze the ability of minimum capital requirements to restore the liquidity provision through the interbank market in a moral risk context. In particular, we study the effectiveness of this type of regulation when liquidity needs can be caused by a deposit shock - a drop in the supply of deposits - or a cash shock-increase in non-performing loans. The methodology used in this work is based on a paper by Macedo $\mathrm{a}$ Troster (2019), which presents a valuable tool to analyze the behavior of the banking industry. Here we extend their work by introducing regulatory capital and study how this regulatory tool affects financial stability. The results show that the capital requirements are a useful tool to reduce the moral risk in the banking industry and, in this way, reestablish the interbank trade. In addition, we show that this type of regulation is more effective in dealing with a cash shock than a deposit shock. Our analysis is relevant in order to achieve financial stability, since it allows us to understand how capital requirements operate in the interbank market.

KEY WORDS: Systemic risk, Capital Requirements, Financial Stability.

PALABRAS CLAVES: Riesgo de liquidez, Requerimientos de Capital, Estabilidad Financiera. 


\section{Introducción}

El sistema bancario es esencial para el funcionamiento de la economía real. Los bancos actúan como intermediarios entre ahorradores e inversores canalizando los recursos de la economía hacia su uso más productivo (Allen et al., 2008). Sin embargo, cualquier falla en la industria bancaria impactará en el corto o mediano plazo en el sector productivo a través de las tasas de interés y la asignación del crédito. Uno de los problemas latentes más comunes en esta industria es el riesgo moral entre sus participantes. Los depositantes no pueden controlar a los bancos, y los bancos no pueden observar cómo los tomadores de préstamos invierten su dinero. Por tal motivo, estas instituciones cuentan con una fuerte supervisión externa con el fin de garantizar su funcionamiento y evitar así problemas en la economía real.

En este documento analizaremos un sector particular de la industria bancaria: el mercado interbancario. Este mercado permite la correcta asignación de liquidez entre entidades bancarias garantizando su correcto funcionamiento y la estabilidad del sistema financiero (Bhattacharya \& Gale, 1987; Allen \& Gale, 2000; Freixas et al., 200o; Huang \& Ratnovski, 2011; Acharya \& Merrouche, 2012; Acharya \& Mora, 2015). Sin embargo, este mercado no está exento de un problema de riesgo moral. Bancos con un alto nivel de préstamos clasificados como dudosos (NPLs), es decir prestamos en los cuales el tomador ha dejado de pagar el principal o los intereses, podrían ocultar pérdidas y ser reacios a desprenderse de este tipo de activos (Sheng,1996; Gunther \& Moore, 2003; Niinimaki,2012). Las entidades bancarias pueden proceder de esta manera con el fin de evitar costos reputacionales (Gabrieli, 2010), fire-sale prices (Diamond \& Rajan, 2011) o incrementos en los requerimientos de capital (Kashyap et al., 2008).

Este comportamiento es posible debido a la asimetría de información entre los participantes del mercado interbancario. Básicamente, los bancos con necesidades de liquidez son capaces de ocultar sus estados patrimoniales cuando los bancos oferentes de liquidez no pueden observar ni la composición ni la calidad de los activos de otras instituciones. Este problema de información asimétrica puede llevar a una contracción total de la oferta de liquidez en el mercado interbancario. Así, bancos solventes con necesidades de liquidez podrían ser incapaces de obtener financiamiento externo cuando son golpeados por shocks de liquidez, lo que puede generar serios problemas en el sector real.

Durante la reciente crisis financiera en Estados Unidos, bancos con una alta proporción de NPLs sufrieron severos problemas para acce- der al mercado interbancario (Brunnermeier, 2009; Huang, 2010; Purnanandam, 2011; Gibson et al., 2018). Sin embargo, los bancos europeos experimentaron problemas de liquidez no solo por el aumento de sus NPLs, sino también debido al fenómeno conocido como "vuelo de depósitos" (Cocco et al., 2009; Connor \& O’Kelley, 2012; Moro,2014; Roman \& Sargu, 2015; Ozili, 2019). Este fenómeno se vio principalmente en países como España, Portugal, Irlanda y Grecia, donde las entidades bancarias fueron afectadas por una reducción en la oferta de depósitos sin precedentes. En consecuencia, los bancos europeos se vieron forzados a incrementar sus tasas de interés para obtener financiación externa, lo que debilitó seriamente sus balances e implicó enormes dificultades para acceder al mercado interbancario (Van Rixtel \& Gasperini, 2013; Dimitrios et al., 2016; Grigorian \& Manole, 2017).

Basados en el hecho de que los bancos son entidades con responsabilidad limitada, Macedo \& Troster (2019) encuentran que shocks de liquidez pueden exacerbar el problema de riesgo moral en el mercado interbancario. Estos shocks son capaces de incrementar el nivel de riesgo del portafolio del banco al reducir los incentivos de estas instituciones a eliminar malos prestamos de su cartera. En particular, los autores muestran que shocks de liquidez originados en el activo bancario son más perjudiciales que aquellos originados en el pasivo como, por ejemplo, una reducción en la oferta de depósitos. Así, incrementos en los NPLs aumentan el riego moral y la chance de una falla del mercado interbancario en mayor proporción que reducciones en la oferta de depósitos.

Es sabido que las autoridades regulatorias pueden atenuar este tipo de comportamiento a través del uso de requerimientos de capital. Furlong \& Keely (1989) encuentran que incrementar los requisitos de capital reduce el incentivo de los bancos a tomar un nivel excesivo de riesgo. Rochet (1992) resalta el hecho de que esta herramienta regulatoria puede tener un efecto dual sobre el riesgo del banco cuando su gerente es adverso al riesgo. Repullo (2004) demuestra que cuando los márgenes de intermediación son pequeños, los requerimientos de capital inducen un comportamiento prudente por parte de los bancos. Hellmann, Murdock \& Stiglitz (2000) encuentran que en un ambiente competitivo las exigencias de capital deben estar acompañadas por topes en la tasa de depósitos para reducir los incentivos bancarios a tomar un excesivo nivel de riesgo. Sin embargo, Genotte \& Pyle (1991) señalan que este tipo de regulación podrían no tener el efecto deseado sobre la estabilidad financiera cuando los bancos escogen de manera endógena el tamaño de su portafolio. Además, Besanko \& Kanatas (1996) establecen que si la industria bancaria es afectada por más de un problema de riesgo 
moral mayores niveles de capital no aseguran un comportamiento prudente por parte de los bancos. Finalmente, el lector puede encontrar una detallada discusión sobre regulación de capital en Allen \& Gale (2003).

Dado que la literatura provee resultados mixtos sobre la efectividad del capital regulatorio, intentamos dar respuestas a las siguientes preguntas. ¿Pueden los requerimientos de capital aminorar el problema de riesgo moral y restaurar el comercio interbancario? ¿Bajo qué escenario este tipo de regulación es eficaz? Para dar respuesta a estos interrogantes utilizaremos la metodología desarrollada en $\mathrm{Ma}$ cedo \& Troster (2019), la cual permite estudiar el comportamiento del mercado interbancario cuando este es afectado por un problema de riesgo moral.

Macedo \& Troster (2019) establecen que la incapacidad del mercado interbancario para proveer liquidez está explicada por la naturaleza del shock que sufrieron los bancos antes que el monto de las necesidades de liquidez generadas por dicho shock. Por lo tanto, el origen de los shocks de liquidez es una importante razón para explicar posibles fallas en el mercado interbancario. Este resultado surge porque, dependiendo de su origen, los shocks de liquidez tendrán un efecto diferente en el balance de los bancos y, por lo tanto, en su comportamiento. La novedad de nuestro trabajo consiste en analizar la efectividad de los requerimientos de capital bajo dos tipos de shock de liquidez, tal como sucedió durante la crisis financiera europea. Entender la conducta de las instituciones bancarias es esencial para el desarrollo de requerimientos de capital óptimos, reestablecer el comercio interbancario $y$, mejorar así, la estabilidad del sistema financiero.

Estructuramos el trabajo de la siguiente manera. En la Sección I se describe la metodología. En la Sección II se formaliza el modelo de una economía de libre mercado, mientras que en la Sección III se presenta el problema de un regulador que utiliza capital regulatorio para maximizar el bienestar social. En la SeCᄀción IV se muestran los principales resultados del modelo con y sin regulación, los cuales se discuten en la siguiente sección. Finalmente, se presentan las conclusiones.

\section{Metodología}

Como mencionamos anteriormente, nuestro análisis normativo está basado en el trabajo de Macedo \& Troster (2019). Estos autores modelizan el mercado interbancario, como proveedor de liquidez, en un contexto de riesgo moral. El problema de riesgo moral ha sido ampliamente estudiado en la literatura (Fundenberg \& Tirole ,1990; Holmstrom \& Tirole, 1997; Holmstrom \& Tirole, 2000; Farhi \& Tirole, 2012). Sin embargo, Macedo \& Troster
(2019) analizan un problema particular de riesgo moral; cómo diferentes shocks de liquidez impactan en el comportamiento de los bancos durante una crisis. En base a esto se definen dos tipos de shocks. El primero es denominado shock de depósitos. Este shock proviene del lado del pasivo bancario cuando los depositantes requieren una mayor tasa de interés para mantener sus ahorros en el sistema bancario. El segundo tipo de shock es denominado shock de caja y surge del activo bancario cuando el flujo de ingresos proveniente de los prestamos se ve reducido. Este tipo de shock intenta capturar el efecto de un incremento en los NPLs en el portafolio de un banco

Un elemento clave de este modelo es que cada banco es monopolista en su respectivo mercado de depósitos, lo que implica que dichos mercados se encuentran aislados. No obstante, los bancos pueden tener incentivos a recurrir al mercado interbancario cuando son afectados por shocks de liquidez. Estos incentivos surgen porque los bancos afectados son capaces de mitigar el efecto de los shocks a través de financiamiento en el mercado interbancario. Sin embargo, este mercado puede verse influenciado por un problema de riesgo moral, dado que los bancos golpeados por un shock podrían no estar dispuestos a remover sus malos prestamos durante una crisis de liquidez. Dicha restructuración financiera trata de restaurar la solvencia incrementando la probabilidad de éxito del portafolio del banco.

Los bancos podrían no llevar a cabo tal restructuración porque pueden ser forzados a vender sus malos activos a fire-sale prices, sufrir incrementos de capital o costos reputaciones. Dado que la decisión de remover los malos prestamos es no observable, el riesgo de cada banco es información privada. Este hecho puede hacer colapsar el mercado interbancario, y así exacerbar el costo de los shocks de liquidez tanto para el banco como para la economía real.

Macedo \& Troster (2019) encuentran que la naturaleza del shock, antes que el monto de las necesidades de liquidez bancarias, explica posibles ineficiencias en la asignación de liquidez por parte del mercado interbancario. En particular, los autores muestran que shocks de liquidez que provienen del activo (incrementos en NPLs) pueden exacerbar en mayor proporción el problema de riesgo moral en el mercado interbancario que shocks provenientes del pasivo (reducción en la oferta de depósitos).

En este documento extendemos el modelo de Macedo \& Troster (2019) con el fin de estudiar la efectividad que tienen los requerimientos de capital para reducir la posibilidad de un colapso interbancario y la chance de una crisis de liquidez. Para ello nos focalizamos en el problema de un regulador cuya función 
objetivo busca maximizar el bienestar social considerando tanto el bienestar de los bancos como el de los depositantes. Así, en busca del máximo beneficio social, el regulador debe determinar los requerimientos mínimos de capital que garanticen un nivel mínimo de estabilidad financiera.

\section{El modelo sin regulación}

En esta sección presentamos las principales características de nuestro sistema bancario cuando no existe ningún tipo de regulación y los agentes se comportan libremente. Para ello, organizamos este apartado en dos subsecciones. En la subsección (a) presentamos la cronología del modelo, mientras que en la subsección (b) describimos detalladamente el problema de los bancos y de los depositantes y la oferta de fondos en el mercado interbancario.

Consideramos un modelo de tres periodos $\mathrm{t}=0,1,2$ donde existen dos bancos, denotados A y B, que son monopolistas en sus respectivos mercados de depósito. Cada mercado de depósitos está habitado por un continuo de idénticos agentes y, por simplicidad, asumimos que no hay factor de descuento entre periodos. En la fecha o, cada banco invierte I unidades de depósitos en un proyecto que produce $C_{-} \mathrm{f}<\mathrm{I}$ y $\mathrm{R}>\mathrm{I}$ en las fechas 1 y 2 , respectivamente. Este proyecto es totalmente ilíquido, lo que implica que su valor de reventa en el momento 1 es cero. Dado que los depósitos deben ser renovados en cada periodo existe un descalce de plazos entre los depósitos (pasivo) y la inversión (activo) del banco.

En el período 1, el Banco A sufre un shock de liquidez el cual puede ser generado por un "vuelo de depósitos" o un incremento en sus NPLs. Dado que solo este banco ve incrementadas sus necesidades de liquidez surgen los incentivos para un mercado interbancario donde, claramente, el Banco B será el encargado de ofrecer liquidez. Sin embargo, un clásico problema de riesgo moral aparece en este mercado porque el Banco A tiene la oportunidad de reestructurar su portafolio removiendo sus malos préstamos. Está acción incrementa la probabilidad de éxito de su inversión, pero no es observable para el Banco B. Así, el funcionamiento del mercado interbancario podría verse afectado debido al nocivo rol de la información asimétrica y riesgo moral.

Modelizamos la oferta de fondos en el mercado de depósitos a través de la existencia de un depositante representativo, el cual vive tres periodos, pero solo consume en la fecha 1 y 2 . Este agente, recibe un ingreso de I en la fecha o y de $\omega$ en el período 1 , mientras que su única fuente de consumo en la fecha 2 serán sus ahorros. Además, consideramos que la única tecnología que tiene este agente para trasladar consumo de un periodo a otro es el depósito bancario y que sus preferencias estarán representadas por la siguiente función de utilidad:

$$
\mathrm{U}\left(\mathrm{c}_{1}, \mathrm{c}_{2}\right)=\frac{\left(\mathrm{C}_{1}^{\theta}\right)}{\theta}+\frac{\left(\mathrm{C}_{2}^{\theta}\right)}{\theta}
$$

donde asumimos $\theta \in(o, 1)$ mide la elasticidad intertemporal de consumo.

\section{Cronología}

Cada banco invierte en un portafolio de préstamos que es totalmente ilíquido. Este portafolio otorgará un pago de Cf en el periodo 1 y un pago de $\mathrm{R}$ o nada en la fecha 2 . La probabilidad de producir un pago de $\mathrm{R}$ difiere entre bancos. El Banco B produce un pago de $\mathrm{R}$ con probabilidad 1. Sin embargo, una vez que el Banco A lleva adelante su inversión descubre que su portafolio está compuesto por una alta proporción de NPLs y, por lo tanto, tiene una chance de obtener R igual a $P_{-} L<1$.

En la fecha 1, el Banco A se ve afectado por un shock de liquidez y, a su vez, tiene la chance de remover los prestamos dudosos de su portafolio. En consecuencia, el Banco A puede mantener su portafolio original, con una probabilidad de éxito de P_L, o puede restructurar su portafolio con el fin de mejorar la calidad de sus préstamos. Está opción implica que la probabilidad de éxito del Banco A pasa de P_L a 1 pero tiene un costo igual a $B$.

Como mencionamos anteriormente, el shock de liquidez puede surgir tanto del lado del activo (shock de caja) o del pasivo (vuelo de depósito) con igual probabilidad. Ambos shocks reducen el valor del banco, pero tienen diferentes efectos sobre su balance. En el evento de shock de caja, el Banco A recibe Cf- $\alpha$ en lugar de Cf en el periodo 1. Por otro lado, cuando el Banco A sufre un shock de depósitos sus costos financieros se incrementan debido a la reducción en la oferta de depósitos. Es importante mencionar que ningún tipo de shock afecta ni el pago en el periodo 3 ni la probabilidad de éxito del proyecto.

En $t=1$, después de que el Banco A es afectado por un shock de liquidez, se abre la oportunidad para la existencia de un mercado interbancario entre ambos bancos. En este mercado se comercian activos líquidos, por ejemplo efectivo, y la cantidad comerciada será denotada con $\mathrm{x}$. Se asume que este mercado es totalmente competitivo, lo que implica que ambos bancos toman la tasa de interés interbancaria R_I como dada. Finalmente, en el período 2 el pago de la inversión bancaria es revelada. Los eventos del modelo son resumidos en el siguiente gráfico. 

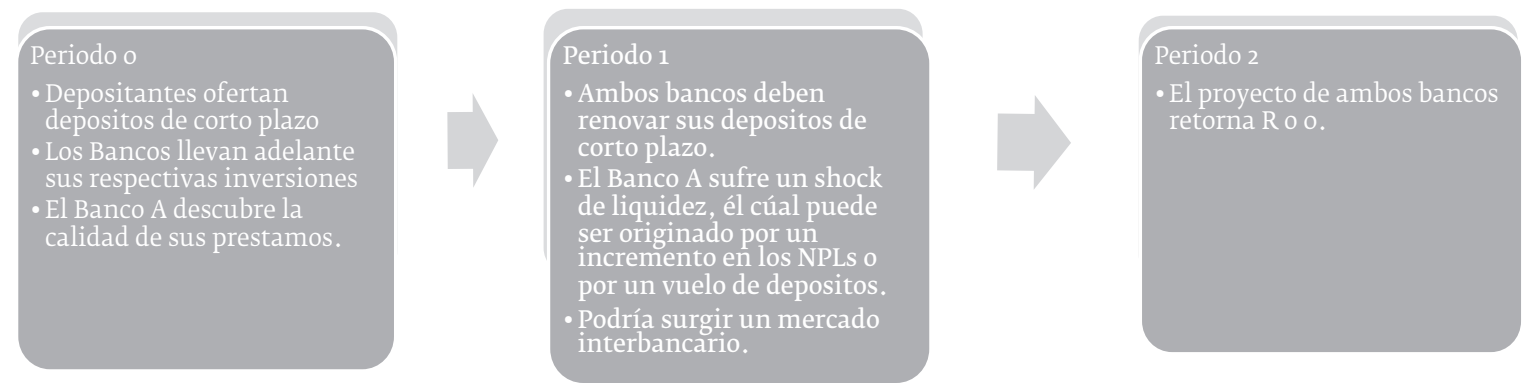

\section{El problema de decisión de los agentes}

El modelo tiene dos estados de la naturaleza indexados por $j=\{C, D\}$, los cuales se refieren a un shock de caja y shock de depósitos respectivamente. En el estado D, los depositantes sufren una reducción de su ingreso y reducen sus ahorros para cubrir sus necesidades de consumo contrayendo la oferta de depósitos en el mercado del Banco A. Por otro lado, si el Banco A sufre una caída en su flujo de caja, el ingreso de los consumidores permanece inalterado. Recuerde que hemos asumido que existen dos mercados de depósitos independientes, uno para cada banco, y que ni el Banco B ni sus depositantes son afectados por los shocks de esta economía

\section{El problema de los depositantes.}

Como vimos anteriormente, las preferencias del depositante representativo están dadas por una función de utilidad con aversión al riesgo constante. Además, este recibe una dotación de I en la fecha o y de $\omega$ en el periodo 1. En consecuencia, este agente debe decidir su senda de consumo y ahorro óptima. La única diferencia entre el mercado de depósito $\mathrm{A}$ y $\mathrm{B}$ es que el deposi $c 2 j=s 1 j \mathrm{r} 1 \mathrm{j}, \mathrm{sa}\left\{\mathrm{c} 1 \mathrm{j}+\mathrm{s} 1 \mathrm{j}=\omega \mathrm{\omega}+\mathrm{I} \theta \theta\left(\mathrm{C}_{2 j}\right.\right.$ ) $+\theta \theta\left(C_{1 j}\right) c t j$, stjmax E tante representativo del mercado A encara dos estados de la naturaleza igualmente probables. En el estado D, sufre un shock de ingresos de tamaño $\rho$, mientras que en el estado C sus ingresos permanecen inalterados. Por lo tanto, este depositante recibe un pago de $\omega_{D}=\omega-\rho$ y $\omega_{C}=\omega$ en los estados $C$ y $D$, respectivamente. Con el fin de que el modelo este bien especificado asumimos que $\rho \in[0, \rho]$. De esta manera, el agente representativo resuelve el siguiente problema

$$
\begin{gathered}
\max _{C_{\mathrm{t} j}, S_{\mathrm{t} j}} E \frac{\left(C_{1 j}^{\theta}\right)}{\theta}+\frac{\left(C_{2 j}^{\theta}\right)}{\theta} \\
\operatorname{sa}\left\{\begin{array}{c}
C_{1 j}+S_{1 j}=\omega_{j}+I \\
C_{2 j}=S_{1 j} r_{1 j},
\end{array}\right.
\end{gathered}
$$

donde $j=\{C, D\}, t=\{0,1\}$ y $r_{t j}$ representa la tasa de interés de los depósitos en el periodo $t$ y estado j. Además, $\Gamma_{t}(s, \omega)$ denota la función inversa de depósitos. Esta función devuelve la mínima tasa de interés que un depositante está dispuesto a aceptar para mantener un monto s de sus ahorros en el banco como depósitos.

\section{ii- El problema del banco $A$}

Ambos bancos necesitan I unidades de fondos para realizar su inversión. Dado que los depositantes no consumen en $\mathrm{t}=\mathrm{o}$, los bancos ofrecen una tasa de interés $r_{0}=1$. En $t=1$, ambos bancos necesitan renovar sus depósitos, pero encuentran óptimo utilizar el ingreso de la inversión $\mathrm{C}_{\mathrm{f}}$ para reducir su nivel de deuda. Así, el monto total de depósitos que los bancos necesitaran renovar en el periodo 1 es igual a $\mathrm{L}=\mathrm{I}-\mathrm{C}_{\mathrm{f}}$.

Ahora bien, el Banco A puede ver incrementadas sus necesidades de liquidez por dos diferentes, y mutuamente excluyentes, razones. En caso de un incremento en sus NPLs, el flujo de caja de la inversión en $\mathrm{t}=1$ es reducido a $\mathrm{C}_{\mathrm{f}}-\alpha$. Así, el banco debe renovar un monto $\mathrm{L}_{\mathrm{C}}=\mathrm{I}-\left(\mathrm{C}_{\mathrm{f}^{-}} \alpha\right)$ de depósitos. Si no existe un mercado interbancario, el costo de renovar este monto de depósitos estará dado por:

$$
\Gamma_{1 c}\left(\mathrm{~L}_{\mathrm{C}}, \omega_{\mathrm{C}}\right) \mathrm{L}_{\mathrm{C}}
$$

En el caso de que los depositantes del Banco A sufran un shock de ingresos $\rho$, la oferta de depósitos de este banco se verá reducida. Sin embargo, el monto de depósitos que este banco necesita no se verá afectado. Es decir, L_D =L. Por lo tanto, el principal efecto de este shock es incrementar el costo de financiamiento del Banco A, el cual estará dado por:

$$
\Gamma_{1 c}\left(\mathrm{~L}_{\mathrm{D}}, \omega_{\mathrm{D}}\right) \mathrm{L}_{\mathrm{D}}
$$


Si el Banco A pueda acceder al mercado interbancario, este puede reducir sus costos sustituyendo parte de los depósitos por préstamos interbancarios. Así, luego de la ocurrencia de un shock tipo por $\mathrm{j}=\{\mathrm{C}, \mathrm{D}\}$, el Banco A debe decidir la combinación optima entre depósitos $\mathrm{y}$ fondos interbancarios $\mathrm{x}$ para minimizar sus costos de financiamiento. Es decir, debe resolver el siguiente problema:

$$
\min _{(x \geq 0)} C_{j}(x) \equiv \Gamma_{1 j}\left(s_{j}^{A}(x), \omega_{j}\right) s_{j}^{A}(x)+R_{I} x,
$$

donde R_I representa la tasa de interés del mercado interbancario. El costo de financiamiento para el Banco $A, C_{-} j^{\wedge} A(x)$, está dado por la suma del costo de obtener un monto $\mathrm{s}_{-}$ $\mathrm{j}^{\wedge} \mathrm{A}(\mathrm{x}) \equiv \mathrm{L}_{-} \mathrm{j}-\mathrm{x}$ de depósitos y el costo de obtener un monto $x$ de fondos interbancarios. Resolviendo el problema de minimización descripto anteriormente, tenemos que la demanda de fondos interbancarios estará definida como $X_{-} j^{\wedge} A\left(R_{-} I\right) \equiv X_{-} j^{\wedge} A$, donde para cada valor de $\mathrm{R}_{-} \mathrm{I}, \mathrm{x}_{-} \mathrm{j}^{\wedge} \bar{A}$ se define implícitamente como:

$$
R_{\mathrm{I}}=\Gamma_{i j}\left(s_{j}^{\mathrm{A}}(\mathrm{x}), \omega_{\mathrm{j}}\right)+\frac{\partial \Gamma_{\mathrm{j}}\left(s_{\mathrm{j}}^{\mathrm{A}}(\mathrm{x}), \omega_{\mathrm{j}}\right)}{\partial s_{\mathrm{j}}^{\mathrm{A}}(\mathrm{x})} \frac{\partial s_{\mathrm{j}}^{\mathrm{A}}(\mathrm{x})}{\partial \mathrm{x})} s_{\mathrm{j}}^{\mathrm{A}}(\mathrm{x})
$$

\section{iii- Equivalencia entre shocks}

Con el objetivo de poder comparar el efecto que ambos tipos de shocks tienen sobre el mercado interbancario, definiremos el shock de caja como función de $\rho$ para asegurarnos que ambos shocks le generan al Banco A la misma necesidad de liquidez. Es decir, mediante esta definición nos aseguramos de que, para cada valor de $\rho$, el banco necesita la misma cantidad de efectivo para continuar con sus operaciones independientemente del tipo de shock.

Para hacer esto, primero determinamos la cantidad de liquidez que necesita el Banco A cuando un shock de tamaño $\rho$ ocurre en el estado D. Luego, definiremos el tamaño de un shock de caja simplemente como:

$$
\alpha(\rho)=\mathrm{L}-S_{1 \mathrm{D}}\left(\hat{\mathrm{r}}_{1}, \omega_{\mathrm{D}}\right),
$$

donde $\hat{S}_{1}=\Gamma_{1 j}$ (L, $\left.\omega\right)$ representa la tasa ofrecida por Banco A para obtener L unidades de depósitos cuando no hay ningún shock. Por lo tanto, $\mathrm{S}_{1 \mathrm{D}}\left(\hat{\mathrm{r}}_{1}, \omega_{\mathrm{D}}\right)$ es el volumen de depósitos ofrecido por los consumidores a la tasa $\mathbb{A}_{1}$ cuando hay un shock tipo D.

\section{iv- La oferta de préstamos interbancarios.}

La posibilidad de recurrir al mercado interbancario ayuda a los bancos a sobrellevar mejor sus problemas de liquidez. En nuestro modelo, el Banco B actúa como prestamista en dicho mercado porque no se ve perjudicado por ningún tipo de shock. Sin embargo, el comercio interbancario podría verse afectado si el Banco A no tiene los suficientes incentivos a restructurar su portafolio y eliminar malos préstamos. Asumiremos que, si el Banco A restructura su portafolio, este siempre tendrá un valor presente positivo dado por:

$$
R-\left(\Gamma_{1 j}\left(L_{j}, \omega_{j}\right) L_{j}+B\right)>0,
$$

donde el primer término es el valor de la cartera de préstamos y el segundo es el costo de financiación y de restructuración del banco en autarquía. Por otro lado, si el Banco A no restructura sus préstamos, el valor presente de su portafolio será negativo y el Banco B perderá dinero en términos esperados si presta en el mercado interbancario. El valor esperado del banco en caso de que este no restructure su portafolio será igual a:

$$
P_{L} R-\Gamma_{1}(L, \omega) L<0,
$$

donde el segundo término representa el costo de financiamiento cuando no hay ningún tipo de shock. Así, el Banco B solo proveerá fondos en el mercado interbancario si la siguiente restricción de compatibilidad es satisfecha:

$$
R-\frac{B}{\Delta P}-\Gamma_{1 j}\left(s_{j}^{A}(x), \omega_{j}\right) s_{j}^{A}-R_{I} x \geq 0,
$$

donde $\Delta P=1-P_{L}$. También definimos $\rho_{D}$ y $\rho_{C}$ como los máximos valores de $\rho$ tal que la restricción de compatibilidad es igual a cero bajo el shock tipo j. Es claro que para todo $\rho>\rho_{D}$ dicha restricción no se cumple bajo un shock de depósito, mientras que si $\rho>\rho_{C}$ el Banco A no tiene incentivos de restructurar su portafolio si un shock de caja ocurre.

El Banco B solo proveerá fondos en el mercado interbancario luego de cumplir con sus obligaciones $\mathrm{L}=\mathrm{I}-\mathrm{C}_{\mathrm{f}}$. En base a esto, el costo neto de proveer fondos en el mercado interbancario estará dado por:

$$
C_{I}^{B}(x) \equiv \Gamma_{1}\left(s_{j}^{B}(x), \omega\right) s_{j}^{B}(x)-\Gamma_{1}\left(s_{j}^{B}(o), \omega\right) s_{j}^{B}(o),
$$

donde $s_{i}(x)=L+x$ representa el monto de depósitos que debe tomar el Banco B. Este monto esta dado por la suma de sus obligaciones $y$ de la cantidad x que desea inyectar en el en el mercado interbancario. Por lo tanto, dada la tasa de interés interbancaria $R_{I}$, el Banco $B$ determina la oferta de fondos resolviendo el siguiente problema:

$$
\min _{(x \geq 0)} R_{I} x-C_{I}^{B}(x) .
$$


La oferta de fondos interbancarios es denotada por $\mathrm{X}_{j}^{\mathrm{B}}\left(\mathrm{R}_{\mathrm{I}}\right) \equiv \mathrm{X}_{\mathrm{j}}^{\mathrm{B}}$, donde $\mathrm{x}_{\mathrm{j}}^{\mathrm{B}}$ está se define implícitamente como:

$$
R_{1}=\Gamma_{1}\left(s^{B}\left(x_{j}^{B}\right), \omega_{j}\right)+\frac{\partial \Gamma_{1}\left(s\left(x_{j}^{B}\right), \omega\right)}{\partial s^{B}\left(x_{j}^{B}\right)} \frac{\partial s\left(x_{j}^{B}\right)}{\partial x_{j}^{B}} s^{B}\left(x_{j}^{B}\right),
$$

y satisface la restricción de compatibilidad definida previamente.

\section{El modelo bajo un regulador}

Por su naturaleza, la industria bancaria es una industria altamente apalancada ya que los bancos llevan adelante sus operaciones con el dinero de los depositantes. Este hecho implica que los bancos pueden tener incentivos a invertir en carteras de préstamos sumamente riesgosas, ya que ellos no internalizan los costos de una posible quiebra. En consecuencia, los reguladores deben dictar ciertos requerimientos y restricciones con el propósito de evitar que los bancos asuman niveles de riesgos excesivamente altos. En esta sección estudiamos el problema de un regulador que utiliza requerimientos de capital con el fin de disminuir el problema de riesgo moral en el mercado interbancario e incrementar la estabilidad financiera.

Para estudiar este problema, consideramos que el regulador requiere que los bancos mantengan un nivel de capital k en el periodo cero. Además, asumimos que el capital tiene un costo de oportunidad y. De esta manera, si el proyecto del banco es exitoso, el banco perderá solo el costo de oportunidad del capital, que estará dado por yk, mientras que si el proyecto fracasa el banco perderá todo su capital. De acuerdo a estas especificaciones, la restricción de compatibilidad bajo requerimientos de $\mathrm{ca}$ pital está dada por:

$$
C C_{j}(\rho) \equiv R+k(1-\gamma)-\frac{B}{\Delta P}-\Gamma_{i j}\left(s_{j}^{A}(x), \omega_{j}\right) s_{j}^{A}-R_{I} x \geq 0,
$$

donde la única diferencia con respecto al caso sin regulación es el termino $\mathrm{k}(1-\mathrm{y})$. Este término captura el mayor incentivo que tienen los bancos a comportarse de manera prudente cuando están sujetos a requerimientos de capital.

De ahora en más, asumiremos que $\rho$ se distribuye de manera uniforme sobre el intervalo $\left[o,\left(\rho^{-}\right]\right.$. Luego, el regulador debe considerar la distribución $\rho$ para determinar el nivel óptimo de $\mathrm{k}$. Al igual que lo hicimos con el modelo sin regulación, definimos $\rho_{C}(k)$ y $\rho_{\mathrm{D}}(\mathrm{k})$ como los máximos valores de $\rho$ tal que la restricción de compatibilidad (14) es satisfecha. Note que cuando $\mathrm{k}=\mathrm{o}$, estamos en el caso sin regulación $y$, por supuesto, $\rho_{-} \mathrm{j}(\mathrm{k})>\rho_{\mathrm{j}}(\mathrm{o})$ para todo $\mathrm{k}>\mathrm{o} \mathrm{y}$ $j=\{C, D\}$.
El beneficio social de utilizar un nivel de capital k cuando un shock tipo j ocurre está dado por la función $\mathrm{BS}_{\mathrm{j}}(\mathrm{k})$, la cual se define formalmente de la siguiente manera:

$$
B S_{j}(k)=\int \frac{\rho_{j}(k)}{p_{j}(0)}\left[R-\Gamma_{j_{j}}\left(s_{j}^{A}(x), \omega_{j}\right) s_{j}^{A}-R_{I} x-B-\left(P_{L} R-\Gamma_{i j}\left(L_{j}, \omega_{j}\right) L_{j}\right)\right] d p-\gamma k .
$$

La integral representa el incremento del producto de la economía debido a la reducción del problema del riesgo moral y la mayor provisión de liquidez por parte del mercado interbancario. Para entender por qué los requerimientos de capital solo son útiles en el intervalo $\left[\rho_{j}(\mathrm{o}), \rho_{\mathrm{j}}(\mathrm{k})\right]$, analicemos lo que sucede fuera de dicho intervalo. Si $\rho \in\left[o, \rho_{j}\right.$ (o) $] U$ $\left[\rho_{j}(k), \bar{\rho}\right]$ los requerimientos de capital no son efectivos. En el intervalo $\left[o, \rho_{j}(o)\right]$ los shocks son pequeños $y$, por lo tanto, el Banco A tiene incentivos de comportarse de manera prudente sin la necesidad de capital regulatorio. En consecuencia, requerimientos de capital no son necesarios porque este banco puede acudir al mercado interbancario para satisfacer sus necesidades de liquidez. En el intervalo $\left[\rho_{\mathrm{j}}\right.$ $\left.(k), \rho^{-}\right]$los shocks son grandes y, por lo tanto, un nivel de capital $k$ no es suficiente para restaurar el mercado interbancario. En este caso, utilizar un nivel de requerimiento k implicaría un costo social neto igual a yk y ningún beneficio para la economía.

Sin embargo, en el intervalo $\left[\rho_{\mathrm{j}}(\mathrm{o}), \rho_{\mathrm{j}}(\mathrm{k})\right]$ un nivel $k$ de capital restaura el comercio en el mercado interbancario. Luego, el incremento en el bienestar social estará dado por el aumento en el valor del banco que surge al comportarse de manera prudente:

$$
R-\Gamma_{i j}\left(s_{j}^{A}(x), \omega_{j}\right) s_{j}^{A}-R_{I} x-B-\left(P_{L} R-\Gamma_{i j}\left(L_{j}, \omega_{j}\right) L_{j}\right) .
$$

Así, si consideramos que la probabilidad de un shock tipo $j=\{C, D\}$ es 0.5 , el regulador escoge su nivel óptimo de requerimientos de capital resolviendo el siguiente problema de optimización:

$$
\max _{(\mathrm{k} \geq 0)} \Pi_{\mathrm{R}}(\mathrm{k})=0.5 \mathrm{BS}_{\mathrm{C}}(\mathrm{k})+0.5 \mathrm{BS}_{\mathrm{D}}(\mathrm{k}),
$$
ros:

donde asumimos los siguientes parámeTabla 1 Parámetros del modelo

\begin{tabular}{lllllll}
\hline$\omega=4$ & $\mathrm{I}=2.25$ & $\theta=0.5$ & $\mathrm{P}_{\mathrm{L}}=0.4$ & $\mathrm{C}_{\mathrm{f}=1}$ & $\mathrm{R}=3$ & $(\bar{\rho})=1.5$ \\
& & & & & \\
& & & & & &
\end{tabular}

En esta sección presentamos las principales implicaciones de nuestro trabajo. Primero, exhibimos los resultados del modelo sin regulación, ya que necesitamos entender cómo los 
agentes se comportan libremente. A continuación, analizamos los efectos de imponer requerimientos de capital en la economía, los cuales contrastaremos con el mercado sin regulación.

\section{i-Mercado sin regulación}

El primer resultado que surge de nuestro modelo es que el origen de un shock de liquidez es determinante para explicar la capacidad del mercado interbancario de proveer liquidez. Esto lo podemos ver de manera numérica en la Tabla 2, la cual presenta las principales variables del modelo cuando el sistema bancario no está sujeto a ningún tipo de regulación. En la primer columna representamos los valores de $\rho$ que, de acuerdo a la Tabla 1 de parámetros, varía entre o y 1.5. En la segunda columna pre- sentamos los valores de $\alpha(\rho)$ tal como hemos especificado en la ecuación (7). Recordemos que la función $\alpha(\rho)$ especifica cual es la necesidad de liquidez del banco para diferentes valores de $\rho$. En particular, asegura que las necesidades de liquidez del banco sean las mismas independientemente del tipo de shock de liquidez.

En la tercer y cuarta columna mostramos la restricción de compatibilidad del Banco A para cada tipo de shock y diferentes valores de $\rho$. Tal como definimos en la ecuación (14), si $C_{-}(\rho)>0$ el Banco A tendrá incentivos a librarse de sus malos préstamos. Por lo tanto, cuando la función $C C_{j}(\rho)$ es positiva, el banco afectado por el shock de liquidez tipo j será capaz de acceder al mercado interbancario y obtener financiación externa.

Tabla 2: Shocks de liquidez y mercado interbancario

\begin{tabular}{|c|c|c|c|}
\hline$\rho$ & $\alpha(\rho)$ & $\mathrm{CC}_{\mathrm{D}}(\rho)$ & $\mathrm{CC}_{C}(\rho)$ \\
\hline $\mathrm{o}$ & $\mathrm{o}$ & $>0$ & $>0$ \\
\hline 0.25 & 0.077 & $>0$ & $>0$ \\
\hline 0.5 & 0.15 & $>0$ & $>0$ \\
\hline 0.72 & 0.23 & $>0$ & $=0$ \\
\hline 1 & 0.32 & $>0$ & $<0$ \\
\hline 1.12 & 0.36 & $=0$ & $<0$ \\
\hline 1.25 & 0.40 & $<0$ & $<0$ \\
\hline 1.5 & 0.48 & $<0$ & $<0$ \\
\hline
\end{tabular}

Podemos ver, a partir de la Tabla 2, que las necesidades de liquidez bancarias $\alpha(\rho)$ son una función creciente de $\rho$. Además, observamos que los incentivos que tiene el Banco A a restructurar su portafolio decrecen con el tamaño del shock de liquidez, así tanto $C_{D}(\rho)$ como $C_{C}(\rho)$ son funciones decrecientes de $\rho$. Los valores $\rho_{D}$ y $\rho_{C}$ que satisfacen $C_{D}\left(\rho_{D}\right)=0$ y $C C_{C}\left(\rho_{C}\right)=0$ son muy importantes, ya que determinan la cantidad máxima de liquidez que el Banco B está dispuesto a ofrecer en el mercado interbancario.

De acuerdo a los parámetros descriptos en la Tabla 1, el máximo shock de tipo $j=\{-$ $C, D\}$ que puede soportar el mercado interbancario cuando no hay requerimientos de capital $(\mathrm{k}=0)$ es igual a $\rho_{\mathrm{C}}=0.72$ y $\rho_{\mathrm{D}}=1.12$. Dado que $\rho_{D}>\rho_{C}$, el mercado interbancario es capaz de proveer una mayor cantidad de liquidez cuando los shocks surgen del pasivo bancario. La razón de este resultado es que, si bien ambos shocks generan la misma necesidad de liquidez, ambos impactan de manera diferente sobre el balance bancario.

En el evento de un shock de depósitos, los depositantes requieren una mayor tasa de interés para mantener su dinero en el banco. Así, el banco afectado por el shock (Banco A) debe pagar mayores tasas, lo que empeora su situación financiera y reduce su patrimonio neto. Por otro lado, en el caso de un shock de caja los ingresos del banco se ven reducidos, lo que lleva a un incrementando en sus obligaciones netas. Además, al igual que en un shock de depósitos, el banco tiene que incrementar la tasa de interés para atraer más depósitos. Estos efectos hacen que un shock de caja sea más dañino para el banco que un shock de depósitos y, por lo tanto, que $\rho_{\mathrm{C}}<\rho_{\mathrm{D}}$.

Dado que $\rho \in[0,1.5]$ se distribuye de manera uniforme, podemos calcular desde la Tabla 2, la probabilidad de que el mercado interbancario no provea liquidez bajo un shock tipo j. Así, el riesgo de una falla del mercado interbancario si un shock tipo j ocurre, se define como

$$
\Omega\left(\rho_{j}\right) \equiv 1-F\left(\rho_{j}\right)
$$

donde $F\left(\rho_{j}\right)$ es la probabilidad de que $\rho$ tome valores menores o iguales a $\rho_{j}$. Por lo tanto, el riesgo que exista una falla del mercado interbancario cuando las necesidades de liquidez están ocasionadas por un shock de depósitos es igual a $\Omega(0.72)=0.52$, mientras que esta probabilidad se reduce a $\Omega(1.12)=0.25$ cuando las necesidades de liquidez son originadas por un shock de depósitos.

En base a los datos observados en la Tabla 2, podemos distinguir tres regiones. La Figura 1 nos muestra que si $\rho \in[0,0.72]$ o, lo 
que es lo mismo, si las necesidades de liquidez del Banco A son menores a $\alpha(0.72)=0.23$, luego este es capaz de obtener liquidez a través del mercado interbancario independientemente del origen del shock. En este rango, los shocks de liquidez son pequeños $y$, por lo tanto, no tienen un gran impacto sobre el balance del banco. En consecuencia, el banco tiene incentivos a restructurar su portafolio y el problema de riesgo moral no afecta la provisión de liquidez por parte del mercado interbancario. Observemos que para todo $\rho \in[0,0.72], C_{j}(\rho)>0$ es positiva para ambos tipos de shocks.

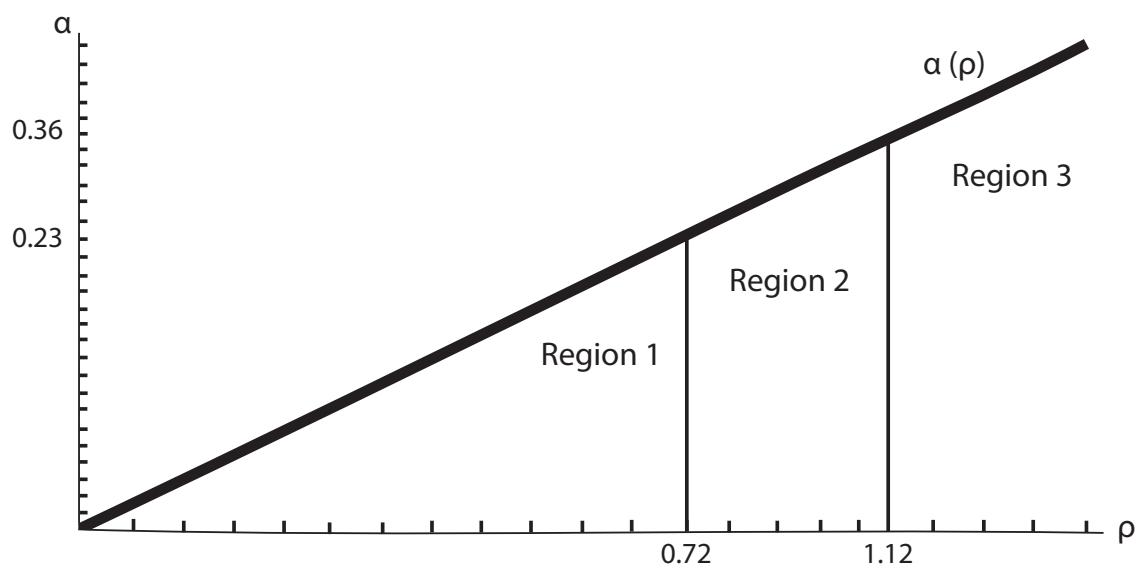

Figura 1 Shock de liquidez y riesgo moral

Si $\rho \in(0.72,1.12]$, el mercado interbancario provee liquidez al Banco A sólo si las necesidades de liquidez están originadas por un shock de depósitos. En el caso de un shock de caja, el mercado no inyectará liquidez, ya que el Banco A no tiene incentivos a restructurar su portafolio. Para todo $p>0.72$, los shocks de caja tienen un fuerte impacto sobre el valor del portafolio bancario. La caída en el valor del portafolio induce al banco a tomar un alto nivel de riesgo para disminuir el impacto de los shocks. El Banco B anticipa este comportamiento y decide no proveer liquidez en el mercado. Finalmente, para todo $\rho>1.12$, los shocks de liquidez son demasiado fuertes independientemente del origen de los mismos. En este caso, el banco siempre encuentra óptimo asumir un alto perfil de riesgo y, por lo tanto, el mercado interbancario será incapaz de proveer liquidez llevando a una asignación ineficiente de recursos en esta economía.

\section{ii- Modelo con regulación}

Veamos ahora cómo cambia la asignación de liquidez en esta economía cuando el regulador introduce requerimientos mínimos de capital. El regulador impone un nivel de capital $\mathrm{k}$ con el fin de reducir los incentivos del banco a incrementar su perfil de riesgo. El principal objetivo de introducir capital re- gulatorio es que el banco internalice parte del costo social de una caída bancaria. Cuanto mayor es el nivel de capital propio invertido, mayor es la pérdida que sufren los accionistas en caso de quiebra. Este hecho induce a los bancos a comportarse de manera prudente y no asumir un perfil de riesgo excesivamente alto.

En la Tabla 3 mostramos como diferentes niveles de requerimientos de capital afectan el bienestar social. Para ello analizamos dos aspectos importantes. El nivel de bienestar en cada estado de la naturaleza y el cambio en la probabilidad de que ocurra una falla en el mercado interbancario cuando existen requerimientos de capital. Como podemos observar, en la primera columna de la Tabla 3 presentamos diferentes valores de capital. En la segunda y tercera columna representamos el bienestar social de utilizar capital bajo un shock de depósitos y un incremento en los NPLs, respectivamente. En la cuarta y quinta columna mostramos el impacto que tienen los requerimientos de capital sobre la probabilidad de una falla del mercado interbancario bajo cada shock de liquidez. Finalmente, en la última columna presentamos el valor esperado del bienestar social de nuestra economía cuando la ocurrencia de un shock tipo $\mathrm{j}=\{\mathrm{C}, \mathrm{D}\}$ es equiprobable, tal como definimos en la ecuación (17). 
Tabla 3 Impacto del capital regulatorio sobre el bienestar social

\begin{tabular}{cccccc}
\hline $\mathrm{k}$ & $\mathrm{BS}_{\mathrm{D}}(\mathrm{k})$ & $\mathrm{BS}_{\mathrm{C}}(\mathrm{k})$ & $\Omega\left(\rho_{\mathrm{D}}\right)-\Omega\left(\rho_{\mathrm{D}}(\mathrm{k})\right)$ & $\Omega\left(\rho_{\mathrm{C}}\right)-\Omega\left(\rho_{\mathrm{C}}(\mathrm{k})\right)$ & $\Pi_{\mathrm{R}}(\mathrm{k})$ \\
\hline 0 & 0.08 & 0.21 & 0 & 0 & 0.15 \\
\hline 0.05 & 0.09 & 0.22 & 0.1 & 0.12 & 0.16 \\
\hline 0.08 & 0.11 & 0.24 & 0.13 & 0.17 & 0.18 \\
\hline 0.11 & 0.10 & 0.28 & 0.18 & 0.21 & 0.19 \\
\hline 0.15 & 0.08 & 0.34 & 0.21 & 0.24 & 0.21 \\
\hline 0.18 & 0.03 & 0.40 & 0.22 & 0.29 & 0.22 \\
\hline 0.21 & -0.01 & 0.50 & 0.24 & 0.31 & 0.25 \\
\hline 0.24 & -0.04 & 0.51 & 0.25 & 0.33 & 0.24 \\
\hline 0.27 & -0.10 & 0.31 & 0.25 & 0.34 & 0.11 \\
\hline 0.3 & -0.12 & 0.22 & 0.26 & 0.34 & 0.05 \\
\hline
\end{tabular}

Desde la Tabla 3 podemos observar que el efecto del capital sobre el bienestar social no es monótono. Tanto el valor de $\mathrm{BS}_{\mathrm{C}}(\mathrm{k})$ como de $\mathrm{BS}_{\mathrm{D}}(\mathrm{k})$ alcanzan un máximo y luego decrecen. En particular, para valores de capital mayores a 0.18 el bienestar social bajo un shock de deposito se torna negativo. Esto se debe al hecho de que imponer requerimientos de capital es socialmente costoso. Luego, si sus beneficios netos son pequeños tendremos un impacto negativo en el bienestar de la sociedad.

Un aspecto importante a tener en cuenta es que existe una relación negativa entre el nivel de capital regulatorio y la probabilidad de un fallo en el mercado interbancario. Es decir, entre mayor es el nivel de capital regulatorio más estable será el sistema financiero. En la tercer y cuarta columna podemos observar que el riesgo de un colapso interbancario disminuye con el nivel de capital bajo ambos tipos de shocks. Esto se debe a que el problema de riesgo moral en el mercado interbancario se hace menos importante a medida que los bancos otorgan prestamos con dinero propio. En otras palabras, la función $C C_{j}(\rho)$ es creciente en $\mathrm{k}$ independientemente del tipo de shock. Observemos desde la ecuación (14) que, en caso de quiebra, los bancos tienen más para perder a medida que el nivel de capital propio aumenta. Finalmente, podemos ver que el bienestar social esperado, el cual está representado en la última columna de la Tabla 2, alcanza un valor máximo para $\mathrm{k}=0.21$. Recordemos que $\Pi_{\mathrm{R}}(\mathrm{k})$ es simplemente el promedio entre la segunda y tercera columna de la Tabla 3 debido a que los shocks tipo D y C son equiprobables.

Ahora analicemos gráficamente el beneficio social de utilizar capital regulatorio. En la Figura 2, las curvas $B S_{j}(k)$, para $j=\{C, D\}$, representan el beneficio social del capital regulatorio cuando un shock tipo j ocurre. En esta figura podemos ver que cualquier nivel de capital k (medido en la abscisa) tiene un mayor retorno social cuando un shock de caja ocurre. Además, la curva $\mathrm{BS}_{\mathrm{C}}(\mathrm{k})$ alcanza su máximo a la derecha de $\mathrm{BS}_{\mathrm{D}}(\mathrm{k})$, lo que muestra que también el retorno marginal de los requerimientos de capital es mayor bajo un shock de caja. Por otro lado, la curva $\Pi_{R}$ (k) representa el beneficio social esperado de utilizar un nivel de capital k cuando la ocurrencia de los shocks es equiprobable. Naturalmente, esta curva se ubica entre las funciones $\mathrm{BS}_{\mathrm{C}}(\mathrm{k})$ y $\mathrm{BS}_{\mathrm{D}}(\mathrm{k})$ y es maximizada en $\mathrm{k}^{\wedge *} \in\left(\mathrm{k}_{\mathrm{D}}^{*}, \mathrm{k}^{*}{ }_{\mathrm{C}}\right)$, donde $\mathrm{k}^{*}{ }_{\mathrm{j}}$ maximiza la función $\mathrm{BS}_{\mathrm{j}}(\mathrm{k})$. Un interesante resultado que surge de nuestro modelo es que, para el nivel $\mathrm{k}^{*}$, $\mathrm{BS}_{\mathrm{C}}\left(\mathrm{k}^{*}\right)>0$ y $\mathrm{BS}_{\mathrm{D}}\left(\mathrm{k}^{*}\right)<\mathrm{o}$. Es decir, el nivel de capital optimo $\mathrm{k}^{\wedge *}$ implica un beneficio social en el caso de un shock de caja y una pérdida social en el caso de un shock de depósitos. Si observamos la Tabla 3, el nivel de capital socialmente óptimo es igual a 0.21 . Así, bajo la regulación óptima tenemos que $\mathrm{BS}_{\mathrm{C}}(0.21)>0$ y $\mathrm{BS}_{\mathrm{D}}(0.21)<0$.

La intuición detrás de este resultado es la siguiente. Como explicamos anteriormente, $\rho_{-} \mathrm{j}(\mathrm{k})$ es una función creciente de $\mathrm{k}$ porque el capital regulatorio relaja la restricción de compatibilidad (14). Esto implica que, a medida que se incrementa el nivel de capital, el problema de información asimétrica se reduce y, por lo tanto, el mercado interbancario es capaz de proveer una mayor cantidad de liquidez. Por otro lado, desde el modelo sin regulación, sabemos que el problema de riesgo moral es menor bajo un shock de depósitos. Así, la oferta de liquidez en el mercado interbancario es mayor bajo un shock de depósitos que bajo un shock de caja. Esto conlleva que se necesitan menores niveles de capital para restaurar el comercio interbancario bajo el primer tipo de shock. Como consecuencia es natural que el capital regulatorio sea más efectivo bajo un shock tipo C y, tenga un retorno marginal negativo, si un shock de depósitos se presenta. 


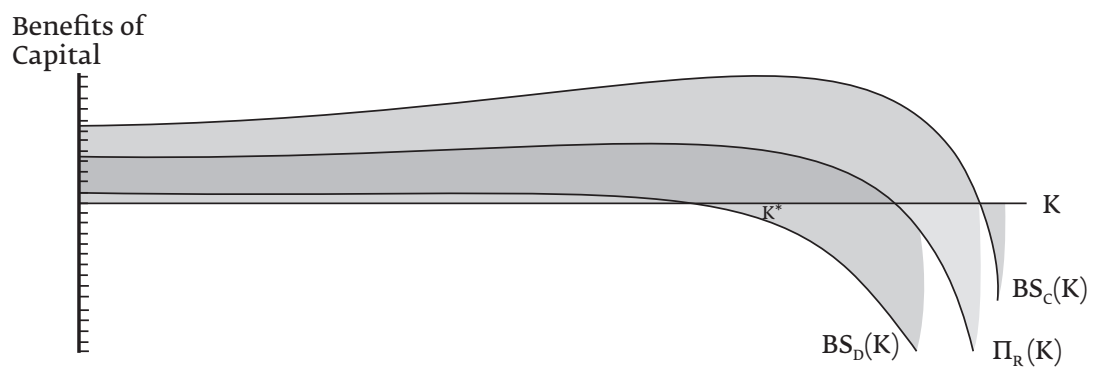

Figura 2: Retorno social del capital regulatorio

Discusión

En el presente documento analizamos la eficacia de los requerimientos de capital para reducir la probabilidad de una crisis de liquidez. Partimos del modelo de Macedo \& Troster (2019), el cual establece que, en un contexto de riesgo moral, el comportamiento de los bancos depende de la naturaleza del shock de liquidez. En base a este resultado, estudiamos si los requerimientos de capital son capaces de inducir un comportamiento menos arriesgado por parte de los bancos y, de esta manera, un mejor funcionamiento del mercado interbancario.

Un elemento clave en nuestro modelo es que los bancos son monopolistas en sus mercados de depósitos y, por lo tanto, esos mercados se encuentran aislados uno del otro. Sin embargo, cuando un shock ocurre los bancos podrían tener incentivos a comercializar fondos a través de un mercado interbancario. En base a los hechos ocurridos durante la última crisis financiera, estas instituciones financieras pueden sufrir problemas de liquidez tanto por un incremento en sus préstamos dudosos como por el fenómeno de "vuelo de depósitos". Luego de ver incrementadas sus necesidades de liquidez, un banco puede acceder al mercado interbancario en busca de financiación. Sin embargo, los bancos afectados por shocks podrían carecer de incentivos a restructurar su portafolio y eliminar los malos préstamos durante una crisis. La restructuración del portafolio busca restituir la solvencia incrementando la probabilidad de éxito de los préstamos. No obstante, los bancos podrían no estar dispuestos a realizar este proceso por varias razones. Primero, ellos podrían verse obligados a vender sus malos préstamos a un precio demasiado bajo. Segundo, estas instituciones financieras podrían sufrir costos reputacionales y, finalmente, el gobierno podría requerir un incremento en sus niveles de capital.

Dado que la decisión de restructurar el portafolio es no observable, el riesgo del banco es información privada y puede afectar seriamente el funcionamiento del mercado interbancario. Así, nuestro modelo resalta la ne- cesidad de que los bancos posean un balance saludable para poder obtener financiamiento desde mercado interbancario. La importancia del balance bancario surge del hecho de que existe una relación negativa entre el valor del patrimonio del banco y su actitud hacia el riesgo. Esta relación es fácil de observar a través de la compatibilidad de incentivos descripta en la ecuación (14). Como podemos ver, a medida que aumenta el pago de los prestamos (R) o disminuyen los costos financieros $\left(\Gamma_{1 j}\left(s_{j}^{A}(x), \omega_{j}\right)\right)$, el banco tendrá mayores incentivos a comportarse de manera prudente incrementando la probabilidad de éxito de su inversión.

Del nuestro modelo surge que, a diferencia de un shock de depósitos, un shock de caja no solo reduce el patrimonio neto del banco, sino que además incrementa sus obligaciones deteriorando el balance bancario más que un shock de depósitos. En otras palabras, un shock de caja es más costoso para el banco que un shock de depósitos. En consecuencia, un banco tendrá menores incentivos a restructurar su portafolio cuando los shocks de liquidez provienen del activo de su balance. Matemáticamente, este resultado surge del hecho de que $\rho_{C}(o)<\rho_{D}(o)$, lo que significa que el mercado está dispuesto a proveer un mayor volumen de liquidez cuando los shocks son originados por una reducción en la oferta de depósitos. Así, la performance financiera de un banco es siempre mayor cuando las necesidades de liquidez se deben a una contracción en la oferta de depósitos. Estos resultados nos muestran que la naturaleza de un shock de liquidez es un factor fundamental para explicar fallas en la distribución de liquidez por parte del mercado interbancario.

El modelo sin regulación nos ha servido para entender cuál es el comportamiento de los bancos y cómo reaccionan estas instituciones a diferentes shocks de liquidez. Ahora discutiremos la efectividad del capital para reducir el problema de riesgo moral en el mercado interbancario. Nuestro modelo contribuye a la literatura de regulación bancaria mostrando que los requerimientos de capital son útiles para restaurar el funcionamiento del mercado interbancario durante una crisis 
de liquidez. El resultado de nuestro trabajo va en concordancia con la literatura clásica que resalta la capacidad del capital regulatorio en reducir problemas de información (Furlong \& Keely,1989; Rochet, 1992; Repullo, 2004; Hellmann, Murdock \& Stiglitz, 200o). Esta rama de la literatura sostiene que los agentes tienden a comportarse de manera prudente cuando invierten capital propio debido al skin in the game effect. Dicho efecto establece que los agentes, bancos en nuestro caso, toman menores riesgos porque invierten su dinero, es decir, se están "jugando su propio pellejo".

Desde la ecuación (14), podemos observar que a medida que aumenta el capital regulatorio la restricción de compatibilidad se relaja, ya que $(1-\gamma)>0$. Así, los incentivos del banco a comportarse de manera prudente crecen con el nivel de capital, lo que implica que $\frac{\left(\mathrm{d} \rho_{\mathrm{j}}(\mathrm{k})\right)}{(\mathrm{d} \mathrm{k})} \geq 0$. Es decir, la cantidad máxima de liquidez que está dispuesto a proveer el mercado interbancario crece con el nivel de capital, lo que reduce el riesgo de liquidez en la economía. Esto es así porque el pago bruto que recibe el banco si no quiebra aumenta con el nivel de capital regulatorio. Observe que $\mathrm{R}+\mathrm{k}(1-\mathrm{\gamma})>\mathrm{R}$, donde $\mathrm{R}$ es el beneficio bruto que obtiene el banco si no hay ningún tipo de regulación. Por lo tanto, cuanto mayor es el nivel de solvencia menor es el riesgo de liquidez en la industria bancaria. Este resultado está plasmado en la Tabla 3, la cual muestra como el capital regulatorio reduce la chance de una falla del mercado interbancario. De esta manera, el capital regulatorio es una herramienta eficaz para reducir problemas de liquidez y mejorar la estabilidad financiera. Esto es así porque un mayor nivel de capital propio hace más costosa la quiebra del banco para los accionistas. Por lo tanto, estos eligen asumir un perfil de riesgo menor. Los bancos con sobrante de dinero anticipan este comportamiento, lo que incrementa su disposición a inyectar liquidez en el mercado interbancario. Así, tenemos una relación inversa entre capital regulatorio y riesgo de iliquidez.

Nuestros resultados contrastan con los trabajos de Besanko \& Kanatas (1996) y Genotte \& Pyle (1991), los cuales demuestran que si existe un problema de riesgo moral multidimensional o los agentes escogen de manera endógena el tamaño de sus portafolios, el capital regulatorio podría tener efectos negativos en la estabilidad financiera. Dado que estos ingredientes no aparecen en nuestro modelo, no podemos afirmar que nuestros hallazgos invalidan los resultados de estos autores. Sin embargo, encontramos que la efectividad del capital varía según la naturaleza del shock de liquidez. Es decir, en términos esperados, la capacidad de los requerimientos de capital para reducir el riesgo moral en el mercado interbancario depende del tipo de shock que ocu- rre en la economía. En particular, el capital tiene un menor retorno social cuando un shock de depósitos ocurre. Observe en la Figura 2 que $\mathrm{BS}_{\mathrm{C}}(\mathrm{k})>\mathrm{BS}_{\mathrm{D}}(\mathrm{k})$ para cualquier nivel de capital. Una manera alternativa de ver este resultado es en términos de probabilidades. Desde el modelo base, sabemos que $\rho_{C}(o)<\rho_{D}(o) y$, por lo tanto, $\rho^{-}-\rho_{D}(0)<\rho^{-}-\rho_{C}(0)$. Dado que $\rho$ se distribuye de manera uniforme sobre el intervalo $\left[o, \rho^{-}\right]$, esta desigualdad implica que los requerimientos de capital serán capaces de actuar con una mayor probabilidad cuando un shock de caja ocurre. Esto hace que el retorno social de utilizar capital regulatorio se más alto bajo este tipo de shock.

\section{Conclusiones}

Debido a que el mercado bancario es una industria altamente apalancada, los reguladores deben dictar ciertos requerimientos, pautas y restricciones con el propósito de evitar que los bancos tomen un riesgo excesivo en sus operaciones. Una herramienta muy utilizada para este propósito son los requerimientos de capital mínimos. Estos requerimientos son impuestos con el fin de mejorar la solvencia del sistema bancario y disminuir el impacto de turbulencias financieras en la economía real.

La crisis financiera europea ha puesto en relieve el rol del mercado interbancario como proveedor de liquidez. Fallas en este mercado conllevan serios problemas al sistema bancario y, en definitiva, al sector productivo que necesita de los bancos para llevar adelante sus operaciones. En este trabajo hemos buscado dar respuesta a los siguientes interrogantes: ¿Pueden los requerimientos de capital aminorar el problema de riesgo moral y restaurar el comercio interbancario? ¿Bajo qué escenario este tipo de regulación es eficaz?

Nuestros resultados establecen que imponer requerimientos de capital a los bancos puede atenuar el problema de riesgo moral en el mercado interbancario. Así, este tipo de regulación reduce la posibilidad de un fallo en dicho mercado y, de esta manera, el riesgo de liquidez de dichas instituciones. Por otro lado, también hemos estudiado bajo qué condiciones el capital regulatorio es más efectivo para restaurar el funcionamiento del mercado interbancario. Si bien los requerimientos de capital mejoran la asignación de liquidez tanto para shocks de liquidez provenientes del activo como del pasivo bancario, hemos encontrado que el capital regulatorio es más eficaz cuando un shock de caja ocurre.

De esta manera, concluimos que imponer requerimientos de capital al sistema bancario ayuda a lograr una mayor estabilidad financiera y menores fluctuaciones en la economía real. Tradicionalmente, el capital regulatorio ha sido pensado para disminuir los problemas 
de solvencia. Sin embargo, nosotros mostramos que el capital regulatorio también puede aminorar problemas de liquidez aumentando la confianza en el mercado interbancario. Este hecho no es trivial, ya que pone de manifestó que requerimientos de capital y liquidez no son herramientas independientes.

\section{Referencias}

Allen, F., Carletti, E., \& Gu, X. (2008). The roles of banks in financial systems. J. Wilson.

Allen F, Gale D (2000) Financial contagion. Journal of Political Economy 108:1-33

Allen F, Gale, D. (2003). Capital adequacy regulation: in search of a rationale. Economics for an imperfect world: Essays in honor of Joseph Stiglitz, 83-109.

Acharya VV, Merrouche O (2012) Precautionary hoarding of liquidity and interbank markets: Evidence from the subprime crisis. Review of Finance $17: 107-160$

Acharya VV, Mora N (2015) A crisis of banks as liquidity providers. The Journal of Finance 70:1-43

Besanko, D., Kanatas, G., 1996. The regulation of bank capital: Do capital standards promote bank safety? Journal of Financial Intermediation 5, 160-183

Bhattacharya S, Gale D (1987) Preference shocks, liquidity, and central bank policy. In: Barnett WA, Singleton K (eds) New Approaches to Monetary Economics: Proceedings of the Second International Symposium in Economic Theory and Econometrics, New York: Cambridge University Press, pp 69-88

Brunnermeier MK (2009) Deciphering the liquidity and credit crunch 2007-2008. Journal of Economic Perspectives 23:77-100

Cocco JF, Gomes FJ, Martins NC (2009) Lending relationships in the interbank market. Journal of Financial Intermediation 18:24-48

Connor G, O'Kelly B (2012) A Coasean approach to bank resolution policy in the Eurozone, available at SSRN: http://dx.doi.org/10.2139/ssrn.2186610

Diamond DW, Rajan RG (2011) Fear of _re sales, illiquidity seeking, and credit freezes. The Quarterly Journal of Economics 126:557-591

Dimitrios A, Helen L, Mike T (2016) Determinants of non-performing loans: Evidence from Euro-area countries. Finance Research Letters 18:116-119

Farhi, E., \& Tirole, J. (2012). Collective moral hazard, maturity mismatch, and systemic bailouts. American Economic Review, 102(1), 60-93.

Freixas X, Parigi BM, Rochet JC (2000) Systemic risk, interbank relations, and liquidity provision by the central bank. Journal of Money, Credit and Banking 32:611-638

Fudenberg, D., \& Tirole, J. (1990). Moral hazard and renegotiation in agency contracts. Econometrica: Journal of the Econometric Society, 1279-1319.

Furlong, F. T. and Keeley, M. C. (1989). Capital regulation and bank risk-taking: A note. Journal of Banking \& Finance, 13(6):883.891.

Gabrieli $S$ (2010) The functioning of the European interbank market during the 2007-08 financial crisis, CEIS Working Paper No. 158. Available at SSRN:http://dx.doi.org/10.2139/ssrn.1525463

Genotte, G., Pyle, D., 1991. Capital controls and bank risk-taking. J. Banking Finance 15, 805-824.
Gibson HD, Hall SG, Tavlas GS (2018) Measuring systemic vulnerability in European banking systems. Journal of Financial Stability 36:279-292

Grigorian D, Manole V (2017) Sovereign risk and deposit dynamics: Evidence from Europe. Applied Economics 49:2851-2860

Gunther JW, Moore RR (2003) Loss underreporting and the auditing role of bank exams. Journal of Financial Intermediation 12:153-177

Hellmann, T. F., Murdock, K. C., \& Stiglitz, J. E. (2000). Liberalization, moral hazard in banking, and prudential regulation: Are capital requirements enough? American economic review, $90(1), 147-165$.

Holmstrom, B., \& Tirole, J. (1997). Financial intermediation, loanable funds, and the real sector. The Quarterly Journal of economics, 112(3), 663691.

Holmström, B., \& Tirole, J. (200o). Liquidity and risk management. Journal of Money, Credit and Banking, 295-319.

Huang R (2010) How committed are bank lines of credit? Evidence from the subprime mortgage crisis. Tech. Rep. 10-25, Federal Reserve Bank of Philadelphia, DOI https://dx.doi.org/10.2139/ ssrn.1659986

Huang R, Ratnovski L (2011) The dark side of bank wholesale funding. Journal of Financial Intermediation 20:248-263

Kashyap A, Rajan R, Stein J (2008) Rethinking Capital Regulation. In: Federal Reserve Bank of Kansas City Symposium on Maintaining Stability in a Changing Financial System, Federal Reserve Bank of Kansas City, Kansas City, pp 431-471

Macedo, Demian and Troster, Victor Emilio, Liquidity Shocks and Interbank Market Failures: The Role of Deposit Flights, Non-Performing Loans, and Credit Market Competition (March 11, 2019). Available at SSRN: https://ssrn.com/abstract $=3350684$

Moro B (2014) Lessons from the European economic and financial great crisis: A survey. European Journal of Political Economy 34:S9-S24

Niinimaki JP (2012) Hidden loan losses, moral hazard and nancial crises. Journal of Financial Stability 8:1-14

Ozili PK (2019) Non-performing loans and financial development: New evidence. The Journal of Risk Finance 20:59-81

Purnanandam, A. (2010). Originate-to-distribute model and the subprime mortgage crisis. The Review of Financial Studies, 24(6), 1881-1915.

Repullo, R. (2004). Capital requirements, market power, and risk-taking in banking. Journal of Financial Intermediation, 13(2):156.182.

Rochet, J.-C. (1992). Capital requirements and the behavior of commercial banks. European Economic Review, 36(5):1137.1170.

Roman A, Sargu AC (2015) The impact of bank-specific factors on the commercial Banks liquidity: Empirical evidence from CEE countries. Procedia Economics and Finance 20:571-579

Sheng A (1996) Banking fragility in the 1980s: An overview. In: Sheng A (ed) Bank Restructuring: Lessons from the 1980s, Washington, D.C.: The World Bank, pp 5-24

Van Rixtel A, Gasperini G (2013) Financial crises and bank funding: Recent experience in the Euro area, BIS Working Paper No. 406. Available at SSRN: https://ssrn.com/abstract $=2229883$ 